\title{
Influence of sheath gas flow rate in Ar induction thermal plasma with Ti powder injection on the plasma temperature by numerical calculation
}

\author{
Yulianta Siregar ${ }^{1,2^{*}}$, Yasunori Tanaka ${ }^{1}$, Tatsuo Ishijima $^{1}$, Yoshihiko Uesugi ${ }^{1}$ \\ ${ }^{1}$ Division of Electrical Engineering and Computer Science, Kanazawa University, Japan. \\ Kakuma, Kanazawa 9201192, Japan. \\ ${ }^{2}$ Departement of Electrical Engineering, University of Sumatera Utara, Indonesia. \\ Jalan Dr. T. Mansur No. 9 Kampus USU, Medan, Indonesia 20155. +6262 8211045
}

\begin{abstract}
Numerical calculation was conducted to study the gas flow fields and temperature distribution in Ar inductively couple thermal plasma (ICTP) with titanium (Ti) powder injection. Influence of sheath gas flow rates at $90 \mathrm{~L} / \mathrm{min}, 80 \mathrm{~L} / \mathrm{min}$ and $70 \mathrm{~L} / \mathrm{min}$ was investigated on the thermal plasma temperature in the torch. Results indicated that higher sheath gas flow rate raises the axial temperature and gas flow velocity in the thermal plasma
\end{abstract}

\section{Introduction}

Inductively coupled thermal plasma (ICTP) in high-pressure and high-power has been widely used for different materials processing such as nanoparticle synthesis [1], thermal barrier coatings [2], fabrication of diamond films [3], surface modification [4], synthesis of fullerene [5]. The ICTP can be an adequate chemical and heat source to different materials processing with little contamination. The ICTP often have high gas temperature and high enthalpy, which can be adequate to evaporate solid feedstock in materials processing. In case of nanoparticle synthesis, the ICTP gives one-step direct processing with rapid evaporation of injected feedstock using the above high gas temperature and high enthalpy. In addition, the ICTP offers rapid cooling of evaporated materials because of high temperature gradient in the downstream portion of the ICTP. This rapid cooling of the evaporated material can enhance nucleation and produce nanoparticles.

The ICTP is usually sustained with sheath gas along the inner plasma torch. The sheath gas is used to supply the plasma gas itself and also to prevent the plasma from contacting the plasma torch from the thermal damage of the torch. It is well known that this sheath gas influence the generation of the ICTP itself, and the temperature and gas flow fields in the ICTP. However, it is not yet enough understood to study the influence of sheath gas flow rate on the temperature field in the ICTP and also on the evaporation rate of feedstock power injected into the ICTP.

\footnotetext{
*Corresponding author: julianta_srg@yahoo.co.id, tanaka@ec.t.kanazawa-u.ac.jp
} 
In this report, the influence of sheath gas flow rate on thermal interactions between argon (Ar) ICTP and titanium (Ti) powder injection was studied using our previously

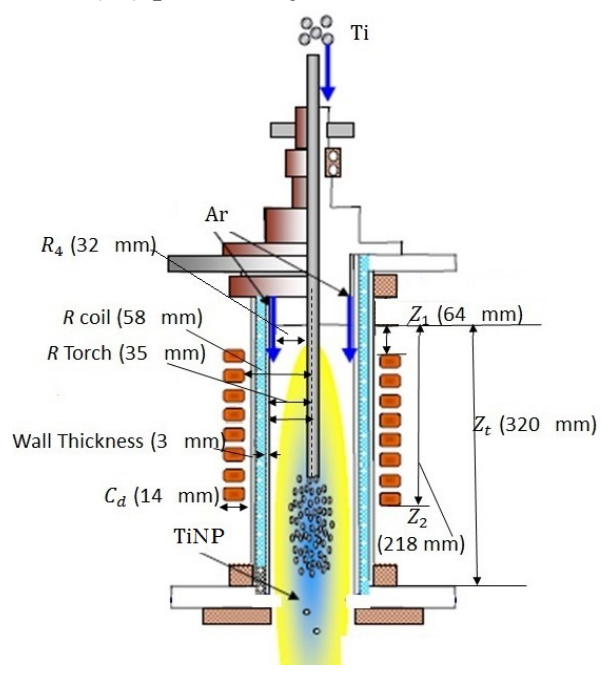

Fig. 1. Configuration of Ar/Ti ICTP torch

developed numerical calculation model [6,7]. Results indicated that higher gas flow rate in the sheath gas enhances evaporation of feedstock by increased thermal plasma temperature along the axis of the plasma torch.

\section{Configuration of inductively coupled thermal plasma torch}

The configuration of the ICTP treated here is shown in Fig. 1. The plasma torch is composed of two coaxial quartz tubes with a length of $320 \mathrm{~mm}$. The inner quartz tube has an internal radius of $35 \mathrm{~mm}$. Around this tube, an 8 turn-coil is wounded with a radius of 58 $\mathrm{mm}$. To this 8 turn-coil, $\mathrm{rf}$ current is supplied to produce electromagnetic field inside the tube. In the tube, the ICTP is sustained by electromagnetic field generated from the coil current. Argon is provided as a sheath gas flow along the inner quartz tube wall. The sheath gas is supplied with a swirl to avoid the plasma from contacting the inner quartz tube.

Titanium feedstock powder is assumed to be injected with Ar carrier gas through a water cooled pipe into the ICTP as shown in Fig.1. For numerical calculation of the temperature and gas flow fields in the ICTP with titanium feedstock powder, a twodimensional cylindrical $r-z$ cross section of this plasma torch was set as a calculation space.

\section{Modeling and calculation of ICTP}

\subsection{Assumptions}

We assumed the following conditions for the ICTP[7]: (i) Local thermodynamic equilibrium (LTE) is established in the ICTP. Thus, all the temperatures including electron temperature and heavy particle temperature are the same to one another. Boltzmann distribution is assumed to the excited state at that temperature. In addition, chemical equilibrium conditions were also set for all reactions. (ii) The optically thin assumption was adopted at wavelengths above $200 \mathrm{~nm}$. (iii) Steady state, axis-symmetric and laminar flow 
were assumed with no viscous dissipation. (iv) The particle-particle interactions were not considered for injected particles. (v) The mean free path of plasma components was assumed to be much smaller than the particle radius. (v) The particle radius was assumed to be much higher than the mean free path of plasma components.(vi) In evaporation, particle always has spherical shape. (vii) The particles are heated uniformly from the around plasmas. Therefore, evaporation uniformly occurs at the particle surface. (viii) Influence of electronic charging on the particle were not considered. (ix) Rocket force was neglected.

Table 1. Titanium powder of thermodynamic properties

\begin{tabular}{|lcc|}
\hline \multicolumn{1}{|c}{ Parameter } & Unit & Value \\
\hline Injection load & $\mathrm{g} / \mathrm{min}$ & 0.5 \\
Mass density & $\mathrm{kg} / \mathrm{m}^{3}$ & 4506 \\
Melting temperature & $\mathrm{K}$ & 1953 \\
Boiling temperature & $\mathrm{K}$ & 3535 \\
Latent heat for melting & $\mathrm{J} / \mathrm{kg}$ & 391000 \\
Latent heat for evaporation & $\mathrm{J} / \mathrm{kg}$ & 8294000 \\
Specific heat of solid & $\mathrm{J} /(\mathrm{kg} . \mathrm{K})$ & 528 \\
Specific heat of liquid & $\mathrm{J} /(\mathrm{kg} . \mathrm{K})$ & 700 \\
Thermal conductivity & $\mathrm{W} /(\mathrm{m} . \mathrm{K})$ & 14 \\
Emissivity of particle source & $(-)$ & 0.4 \\
\hline
\end{tabular}

\subsection{Numerical calculation condition}

In this calculation, the operating frequency for the rf coil current was fixed at $450 \mathrm{kHz}$. The pressure condition was set at 300 Torr $(=40 \mathrm{kPa})$ inside the plasma torch. The input power was set at $40 \mathrm{~kW}$. Sheath gas flow rate was set to three values of $90 \mathrm{~L} / \mathrm{min}, 80 \mathrm{~L} / \mathrm{min}$, and $70 \mathrm{~L} / \mathrm{min}$. To this ICTP, titanium feedstock powder is injected through a water-cooled pipe inserted on the torch axis from the plasma torch head. The injected titanium feedstock powder has a mean diameter of $2 \mu \mathrm{m}$ and a deviation of $0.5 \mu \mathrm{m}$. In the calculation, 35 trajectories for feedstock particles with 7 different diameters with 5 different initial positions were assumed to be injected. The titanium feedstock feed rate was set to 0.5 $\mathrm{g} / \mathrm{min}$. In addition, transport properties and thermodynamic of argon and titanium vapor were in advance calculated as a function of temperature. For solid and liquid thermal properties of feedstock, the values were used in the literatures as indicated in Tab.1.

\section{Calculation results}

\subsection{The temperature distribution of the ICTP}

Figure 2(a), (b) and (c) show the temperature distribution of the ICTP with an sheath gas flow rate of $90 \mathrm{~L} / \mathrm{min}, 80 \mathrm{~L} / \mathrm{min}$ and $70 \mathrm{~L} / \mathrm{min}$, respectively. In case of Fig.2(a) with a sheath gas rate of $90 \mathrm{~L} / \mathrm{min}$, the ICTP has high temperature area about $11000 \mathrm{~K}$ in wide region around radial positions of $10 \mathrm{~mm}<r<30 \mathrm{~mm}$ and axial positions $140<z<235 \mathrm{~mm}$. In this high temperature area, the rf eddy current flows inside the ICTP to heat it. At a sheath gas flow rate of $80 \mathrm{~L} / \mathrm{min}$ as shown in Fig.2(b), the high temperature area at temperatures above $11000 \mathrm{~K}$ becomes smaller around radial positions of $9 \mathrm{~mm}<\mathrm{r}<29 \mathrm{~mm}$ and axial positions of $135 \mathrm{~mm}<\mathrm{z}<225 \mathrm{~mm}$ than that in Fig.2(a). Furthermore, lower sheath gas flow rate at $70 \mathrm{~L} / \mathrm{min}$ case as indicated in Fig.2(c) presents a further smaller high temperature area at temperatures above $11000 \mathrm{~K}$ around radial positions of $15 \mathrm{~mm}<\mathrm{r}<28 \mathrm{~mm}$ and axial positions of $130 \mathrm{~mm}<\mathrm{z}<220 \mathrm{~mm}$. This means that the high temperature area is shifted to upstream with decreasing sheath gas flow rate. This is due to reduced gas flow 
convection transport by decreasing gas flow rate. In addition, the high temperature area is shrunk in axial direction with decreasing gas flow rate. These features can be seen for midtemperature area of $6000-6500 \mathrm{~K}$.

On the axis of the torch, the temperature is higher at higher gas flow rate of $90 \mathrm{~L} / \mathrm{min}$ than at lower gas flow rate of $70 \mathrm{~L} / \mathrm{min}$. This may be because at higher gas flow rate of 90 $\mathrm{L} / \mathrm{min}$, the high temperature area is present near the center axis by strong gas flow around the torch wall. That stronger gas flow make the high temperature plasma present near the axis. This higher temperature around the axis is related to the evaporation rate of feedstock as described later.

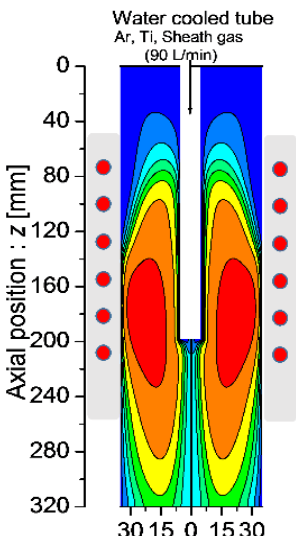

(a)

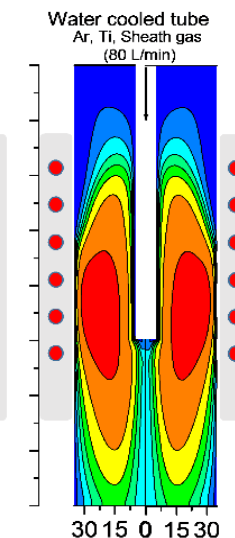

(b)

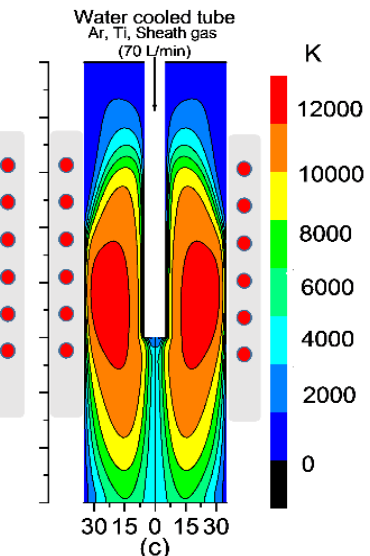

(c)

Radial position : $r[\mathrm{~mm}]$

Fig. 2. The temperature distribution of Ar and Ti powder injection with sheath gas flow rate at $90 \mathrm{~L} / \mathrm{min}, 80 \mathrm{~L} / \mathrm{min}, 70 \mathrm{~L} / \mathrm{min}$.

\subsection{Titanium feedstock particle evaporation}

In this temperature fields of the ICTP, titanium feedstock particles were assumed to be injected. Figure 3 indicates variation in diameters of 15 particles with 5 different initial diameters injected from 3 different initial positions into the sheath gas as a function of axial position. Figure 3(a) and 3(b) corresponds to the results for sheath gas flow rates of $90 \mathrm{~L} / \mathrm{min}$ and $70 \mathrm{~L} / \mathrm{min}$, respectively.

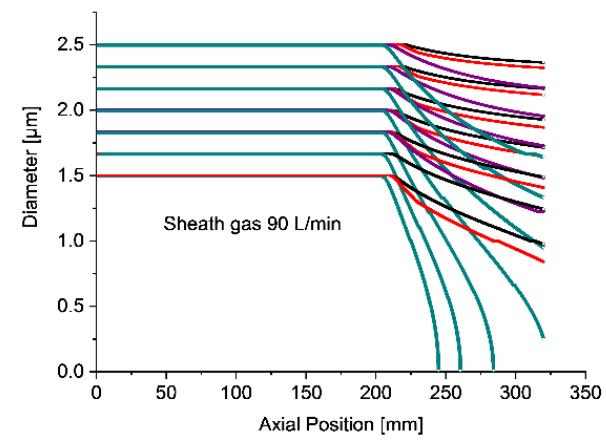

(a)

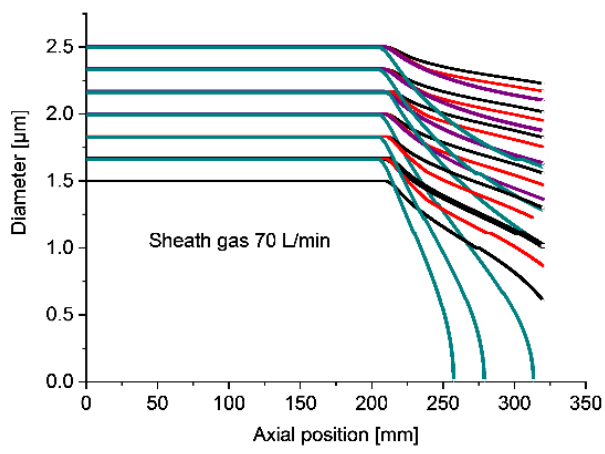

(b)

Fig. 3. Diameter variation of titanium particles injected for 35 kinds with 5 different initial positions and different 7 initial diameters as a function of axial position in argon ICTPs with sheath gas flow rate at $90 \mathrm{~L} / \mathrm{min}$ (a) and $70 \mathrm{~L} / \mathrm{min}$ (b).

The diameters do not change at axial position from 0 to $200 \mathrm{~nm}$ because all the particles 
injected are present inside the water-cooled pipe. From axial position of $200 \mathrm{~mm}$, the particles start flying into the ICTP, and then they are heated by the ICTP. After some fly to be heated, the Ti particles have the diameter decreased because of its evaporation. In this calculation, the particles with initial diameter of $1.5 \mu \mathrm{m}$ are strongly heated from the ICTP, and then the particle diameter decreases to $0.01 \mu \mathrm{m}$. As shown in Fig.3(a) and (b), higher sheath gas flow rate of $90 \mathrm{~L} / \mathrm{min}$ involves rapid decrease in diameter than $70 \mathrm{~L} / \mathrm{min}$. This is attributed to the higher temperature along the torch axis as indicated in the previous section. This evaporation of the feedstock particles produces titanium vapor to the ICTP, which can change the properties of the Ar ICTP.

\subsection{Mass fraction of titanium vapor}

Influence of sheath gas flow rate on titanium vapor creation in the ICTP can be seen in Fig. 4. As shown in this figure, the mass fraction of titanium (Ti) vapor is distributed from the tip of the water-cooled pipe.

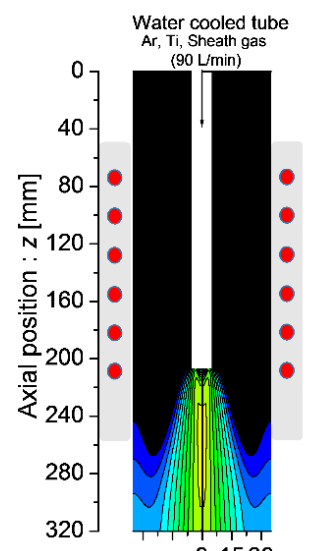

(a)

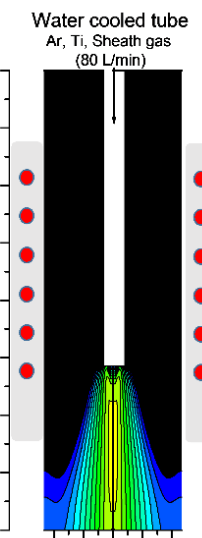

301501530 (b)

Radial position : $r[\mathrm{~mm}]$

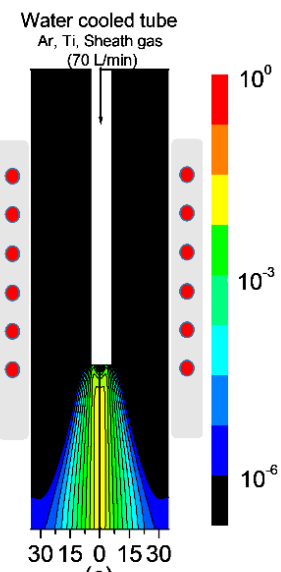

(c)

Fig. 4. Mass fraction of Ar and Ti powder injection with sheath gas flow rate at $90 \mathrm{~L} / \mathrm{min}, 80 \mathrm{~L} / \mathrm{min}$, $70 \mathrm{~L} / \mathrm{min}$

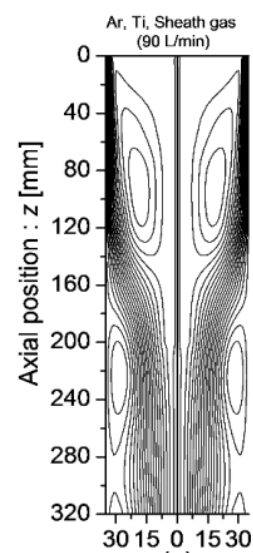

(a)

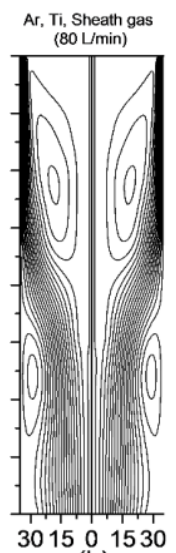

(b)

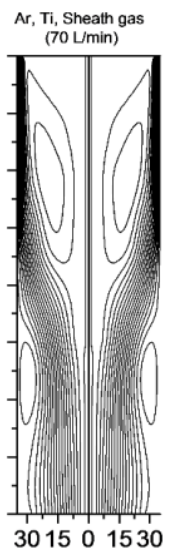

(c)

Radial position : $r[\mathrm{~mm}]$

Fig. 5. Streamline of Ar and Ti powder injection with sheath gas flow rate at $90 \mathrm{~L} / \mathrm{min}, 80 \mathrm{~L} / \mathrm{min}$, $70 \mathrm{~L} / \mathrm{min}$ 
The injected Ti feedstock particles are injected to the ICTP through the feeding pipe. The Ti particles are evaporated during their flight to produce Ti vapor as contamination to Ar ICTP. This contaminated Ti vapor is transported by gas flow convection of Ar carrier gas and by diffusion. This Ti vapor is present downstream of the feeding pipe along the axis. Titanium vapor concentration just slowly increases with decreasing sheath gas flow rate. This is due to lower gas flow velocity along the axis, and thus the Ti vapor concentration is higher at $50 \mathrm{~L} / \mathrm{min}$ of sheath gas flow rate.

Another difference in $\mathrm{Ti}$ vapor concentration is that $\mathrm{Ti}$ vapor is present around the wall at higher gas flow rate of $90 \mathrm{~L} / \mathrm{min}$. This arises from the vortex near the wall. Figure 5 shows the streamline inside the ICTP at different sheath gas flow rate at $90 \mathrm{~L} / \mathrm{min}, 80 \mathrm{~L} / \mathrm{min}$ and $70 \mathrm{~L} / \mathrm{min}$. As indicated in Fig.5, increasing sheath gas flow rate gives strong vortex at the upper side of the plasma torch at axial position $40 \mathrm{~mm}<z<140 \mathrm{~mm}$. Furthermore, another vortex present at axial position $200 \mathrm{~mm}<z<280 \mathrm{~mm}$ around the torch wall is strengthened by increasing sheath gas flow rate. Because of this vortex, the mass fraction of Ti vapor is transported to upstream direction near the torch wall around axial position 240 $\mathrm{mm}<\mathrm{z}<320 \mathrm{~mm}$.

\section{Conclusion}

Numerical simulation was made to study influence of sheath gas flow rate on the temperature distribution in argon (Ar) induction thermal plasma with titanium (Ti) feedstock powder. The calculated temperature distributions of argon thermal plasma and titanium feedstock powder were compared with different sheath gas flow rate at $90 \mathrm{~L} / \mathrm{min}$, $80 \mathrm{~L} / \mathrm{min}$ and $70 \mathrm{~L} / \mathrm{min}$. Results indicated that higher sheath gas flow rate raises the axial temperature distribution and gas flow velocity in the thermal plasma torch.

\section{Acknowledgment}

The authors thank to Kanazawa University, Japan and Ministry of Research, Technology and Higher education (KEMRISTEKDIKTI) of Indonesian Government for the existing bilateral academic-cooperation. An appreciation is also granted to Indonesia Endowment Fund for Education (LPDP) that provides fund through a BUDI-LN Scholarship Scheme.

\section{References}

1. L. Tong and R.G. Reddy. Mater. Res. Bull, 4, 2303 (2006)

2. J. Mao, M. Liu, C.G. Deng, C.M. Deng, K.S. Zhou, Z.Q. Deng, J.Eng. Mater. Technol, 139, 041003 (2017)

3. S. Gottlieb, N. Wohrl, S. Schulz, V. Buck, SpringerPlus, 5, 568 (2016)

4. W. Chen, Y. Xu, S. Shi, Y. Cao, M. Chen, X. Zhou, Scien. Report, 8, 2279 (2018).

5. K.S. Kim, A. Moradian, J. Mostaghimi, G. Soucy. Plasma Chem, 30, 91-110 (2010)

6. Y. Tanaka, T. Sakuta, J. Phys. D: Appl. Phys, 58, 2149 (2002)

7. Y. Siregar, N. Kodama, Y. Tanaka, T. Ishijima, Y. Uesugi, IOP Conf. Series: Materials Scence and Engineering, 309, 012090 (2018) 